\title{
FUNGI OF THE STELLENBOSCH DISTRICT AND IMMEDIATE VICINITY.
}

\author{
By Dr. P. A. van Der BiJl.
}

(From the Department of Phytopathology and Mycology, University of Stellenbosch.)

No separate list of the fungi occurring in the Stellenbosch district has so far been published.

For some years Miss A. V. Duthie has been collecting principally the Myxomycetes and the Higher Basidiomycetes occurring in the district, and I am indebted to her for kindly placing her collection at my disposal and for allowing me to embody it in the present paper.

The only way we can hope to learn the distribution of the South African fungi is to study closely those occurring over small areas, and to extend such work throughout the Union. More lasting benefit is to be derived from the close study of the fungi occurring over limited areas than collecting a few here, there, and everywhere.

In this connection Miss A. Pegler of Kentani rendered valuable service to the study of South African Mycology by giving close attention to the fungi occurring in her district.

In $1898 \mathrm{~J}$. Medley Wood published a list of the fungi occurring in Natal. Incomplete as this list is, it is nevertheless of great assistance to anyone working on fungi in Natal.

In the present list the genera and species are listed alphabetically under the families to which they belong, and the host is also often stated. The Agariceae are omitted from this list. They will require a few seasons to work up satisfactorily, and will be dealt with later. In the case of specimens not collected by myself the name of the collector is always given. The list contains 98 genera, 148 identified species, and 20 fungi not specifically identified. As the study of the fungi of the district progresses the number will no doubt be considerably increased.

With the co-operation of students and friends it is hoped to work up the fungus flora of other districts as well, and to publish these from time to time. 


\section{Myxomycetes.}

Plasmodiophorales.

Plasmodiophora brassicae, Wor., Brassica oleracea.

Myxogastrales.

Ceratiomyxaceae.

Ceratiomyxa-fruticulosa, Macbr. Decayed wood. A. V. Duthie, June 1917.

\section{Physaraceae.}

Badhamia utricularis, Berk. Dead wood. A. V. Duthie, June 1917.

Diachaea leucopoda, Rost. Decayed leaves. A. V. Duthie, Aug. 1916.

Diderma effusum, Morg. A. V. Duthie.

Fuligo septica, Gmelin. Decayed wood.

Leocarpus fragilis, Rost. Dead needles of Pinus. A. V. Duthie, Aug. 1916.

Physarum auriscalpum, Cke. Dead needles of Pinus. A. V. Duthie, Aug. 1916.

Physarum pusillum, Lister. Dead stem, Solanaceae. A. V. Duthie.

Physarum sinuosum, Weinm. A. V. Duthie.

Physarum vernum, Somm. A. V. Duthie.

Didymiaceae.

Didymium difforme, Duby. A. V. Duthie.

Didymium nigripes, var. xanthopus, Lister. A. V. Duthie.

\section{Stemonitaceae.}

Comatricha nigra (Pers.), Schroet. A. V. Duthie.

Lamproderma scintillans, Morgan. On moss. A. V. Duthie.

Stemonitis splendens, Rost. Dead wood. A. V. Duthie.

Stemonitis splendens, Rost., var. Webberi, Lister. A. V. Duthie.

\section{Heterodermaceae.}

Cribraria argillacea, Pers. Decayed stump, Pinus. A. V. Duthie, May 1917.

Dictydium cancellatum, Macbr. A. V. Duthie.

\section{Reticulariaceae.}

Dictydiaethalium plumbeum, Rost. Dead wood. A. V. Duthie.

Reticularia lycoperdon, Bull. Dead wood. A. V. Duthie.

\section{Lycogalaceae.}

Lycogala epidendrum, Fr. Dead wood. A. V. Duthie.

Lycogala flavo-fuscum, Rost. A. V. Duthie. 


\section{Arcyriaceae.}

Arcyria cinerea (Bull.), Pers., Forma digitata. Dead wood. A. V. Duthie. Arcyria denudata, Sheldon. Dead wood. A. V. Duthie.

Arcyria nutans, Grév. Dead wood. A. V. Duthie.

Arcyria Oerstedtii, Rost. Dead wood. A. V. Duthie.

\section{Schizomycetes.}

Actinomyces scabies (Thax.), Guss., Solanum tuberosum.

Bacterium nectarophilum, Doidge, Pirus communis.

Bacterium tumefaciens, Sm., Pirus malus.

Pseudomonas pruni, Sm., Prunus persica, Nov. 1921 ; Prunus domestica (Prunes), Jan. 1922.

\section{Phycomycetes.}

Albuginaceae.

Albugo candida, Lér., Cochlearia Armourica, Dec. 1921 ; Raphanus raphinastrum.

\section{Entomophthoraceae.}

Empusa Grylli (Fres.), Nowakowski.

Empusa muscae, F. Cohn.

\section{Ascomycetes.}

\section{Exoascaceae.}

Taphrina aurea, Fr., Populus pyramidalis, Dec. 1921.

Taphrina deformans (Fcl.), Tul., Prunus persica (Peach and nectarines).

\section{Helvellaceae.}

Morchella conica, Pers. On ground. A. V. Duthie.

\section{Pezizaceae.}

Lachnea hemisphaerica (Wigg.), Gill. A. V. Duthie.

Lachnea sp. A. V. Duthie.

Peziza spp. Two large species and several smaller ones. A. V. Duthie.

\section{Mollisiaceae.}

Aspergillus niger, Bref., Allium cepa. Nov. 1921.

\section{Erysiphaceae.}

The fungi of this family were only found in their conidial fructifications. Their identification was hence based on the hosts on which they occurred.

Erysiphe cichoracearum, D. C., Cucumis pepo., Nicotiana tabacum.

Erysiphe polygoni, D. C., Pisum sativum. Nov. 1921. 
Podosphaera leucotricha (Ell. and Ev.), Salmon Pirus malus. Nov. 1921.

Sphaerotheca pannosa (Wallr.), Lév., Rosa sp. Nov. 1921.

Uncinula necator (Schwein.), Burr, Vitis vinifera.

\section{Hypocreaceae.}

Claviceps paspali, S. \& H., Paspalum. Feb. 1922.

Claviceps sp., Pennisetum macruorum.

\section{Dothidiaceae.}

Phyllachora cynodontis (Sacc.), Niess., Cynodon dactylon. Feb. 1922.

Phyllachora melianthi (Thuem.), Sacc., Melianthus major. Oct. 1922.

Phyllachora sp., Pennisetum macruorum. March 1922.

\section{Coryneliaceae.}

* Corynelia uberata, Ach., Podocarpus. Oct. 1921.

Mycosphaerellaceae.

Mycosphaerella fragariae (Tul.), Linn., Fragaria vesca.

\section{Pleosporaceae.}

Venturia inaequalis (Cke.), Aderh. (Fusicladium stage), Pirus malus.

Venturia pirina, Aderh. (Fusicladium stage), Pirus communis.

\section{Melanconidaceae.}

Calospora arausiaca (Fab.), Sacc., Quercus sp.

\section{Xylariaceae.}

Hypoxylon sp. Old wood. A. V. Duthie.

Xylaria sp. Old wood. A. V. Duthie.

\section{Ustilaginaceae.}

\section{BASIDIOMYCETES.}

Ustilago avenae (Pers.), Jens., Avena sativa. Nov. 1921.

Ustilago bromivora (Tul.), F. v. M., Bromus unioloides. Oct. 1921.

Ustilago hordei (Pers.), K. \& S., Hordeum vulgare. Sept. 1921.

Ustilago maydis (D. C.), Tul., Zea Mays.

Ustilago nuda (Jens.), K. \& S., Hordeum vulgare. Oct. 1921.

\section{Tilletiaceae.}

Tilletia laevis, Ruhn., Triticum vulgare.

Urocystis tritici (Bjerk.), Wint., Triticum vulgare. Oct. 1921.

\section{Melampsoraceae.}

Melampsora tremulae, Tul., Populus sp. Sept. 1921. 


\section{Pucciniaceae.}

Aecidium resinicolum, var. tumefaciens, Wint., Raphria angulata. Oct. 1911.

Phragmidium subcorticum, Wint., Rosa sp. Nov. 1921.

Phragmidium violaceum (Schultz), Wint., Rubus Vitifolius.

Puccinia chrysanthemi, Roze., Chrysanthemum sp.

Puccinia graminis, Pers., Avena sativa, Hordeum sativum, Triticum vulgare.

Puccinia granularis, K. \& Cke., Pelargonium sp.

Puccinia hypochaeridis, Oud., Hypochaeris radicata. Oct. 1921.

Puccinia malvacearum, Mont., Malva parviflora. Oct. 1921. Mathiola sp.

Puccinia maydis, Bereng., Zea Mays. Feb. 1922.

Puccinia phragmites (Schum.), Koern., Phragmites communis.

Uredo Fici, Cast., Ficus sp.

Uromyces betae (Pers.), Kuhn., Beta vulgaris sp. 1921.

Uromyces cyperi, P. Henn., Cyperus sp. Oct. 1921.

Uromyces fabae (Pers.), de B., Vicia Faba. Oct. 1921.

Uromyces romuleae, n. sp., P. v. d. B. (MSS. name), Romulea rosea.

Uromyces striatus, Schroet., Medicago sativa.

Tranzschelia punctata (Pers.), Arth., Amygdalus communis; Prunus armeniaca; Prunus domestica (Prunes); Prunus persica.

\section{Tremellaceae.}

Exidia purpureo-cinerea, Kalch. Dead branch, Quercus, Sept. 1921. Old log, Oct. 1921.

Tremella sp. A. V. Duthie.

Tremella microspora, Lloyd. A. V. Duthie.

\section{Dacromycetaceae.}

Dacromyces deliquescens (Bull.), Duby. Old log. A. V. Duthie.

Hormomyces aurantiacus, Bon. Logs, Quercus. A. V. Duthie.

\section{Thelephoraceae.}

Stereum hirsutum (Willd.), Pers. Common saprophyte on logs of Quercus and other plants.

Stereum purpureum, Pers. Old logs Quercus. Aug. 1922.

Stereum vellereum, Berk. Old logs Quercus.

Thelephora intybacea (Pers.), Fr. In plantations, Pinus.

Thelephôra penicillata, Lloyd. A. V. Duthie.

\section{Polyporaceae.}

Lenzites betulina, Linn. Stump, Quercus. Aug. 1921.

Lenzites Quineensis, Fr. (form of above). Old logs. Oct. 1921.

Merulius lachrymans, Facq. A. V. Duthie. 
Trametes protea, Berk. Stump and logs of Pinus, Populus, and Quercus. Trametes trabea, Otth. Logs of Pinus.

Polyporus adustus, Willd. Stumps Quercus. Aug. 1921.

Polyporus conchatus, Lloyd. Stump Populus.

Polyporus occidentalis, Klotz. Old logs. A. V. Duthie.

Polyporus ochraceus, Pers. Old logs. A. V. Duthie.

Polyporus rusticus, Lloyd. Log Pinus.

Polyporus sanguineus (Linn.), Fr. Old logs. A. V. Duthie.

Polyporus sulphureus (Bull.), Fr. Wound parasite of Quercus. Burned stump of Eucalyptus.

Polyporus versicolor (Linn.), Fr. Wound parasite of Prunus persica. Common saprophyte on various old logs.

Polyporus zonatus, Fr. A. V. Duthie.

\section{Boletaceae.}

* Boletus edulis, Bull. On ground.

Boletus, n. sp. (Herb. No. 508). On ground.

\section{Agaricaceae.}

Amanita muscaria (Linn.), Pers. On ground. A. V. Duthie.

Amanita phalloides (Fr.), Quél. On ground. A. V. Duthie.

Lentinus Lepideus, Fr. Stump of Pinus.

Schizophyllum commune Fr. Wound parasite on Prunus persica and Acacia saligna. Common saprophyte on logs of various trees.

\section{Phallaceae.}

Anthurus Mac Owani, Marl. On ground. A. V. Duthie.

Phallus sp. On ground. A. V. Duthie.

Hymenogastraceae.

Rhizopogon luteolus, Fr. On ground. A. V. Duthie.

Rhizopogon rubescens, Tul. On ground.

Rhizopogon sp. On ground. A. V. Duthie.

\section{Hysterangiaceae.}

Protubera africana, Lloyd. On ground. A. V. Duthie.

\section{Lycoperdaceae.}

Calvatia lilacina, Berk. On ground. A. V. Duthie.

Catastoma magnum. On ground. A. V. Duthie.

Geaster plicatilis, Berk. On ground.

Lycoperdon pratense, Pers. On ground. A. V. Duthie.

Nidulariaceae.

Cyathus vernicosus (Bull.), D. C. On ground. A. V. Duthie. 


\section{Podaxaceae.}

Podaxon carcinomalis, Fr. On termite nest. A. V. Duthie.

\section{Sclerodermataceae.}

Arachnion album, Schw. On ground. A. V. Duthie.

Arachnion scleroderma, Lloyd. On ground. A. V. Duthie.

Polysaccum crassipes, Fr. On ground under Eucalyptus trees.

Scleroderma cepa, Pers. On ground. A. V. Duthie.

Scleroderma laeve, Lév. On ground. A. V. Duthie.

Scleroderma flavidum, Ellis. On ground. A. V. Duthie.

Scleroderma tenerum, Berk. On ground. A. V. Duthie.

\section{Tulostomataceae.}

Tulostoma cyclophorum, Lloyd. On ground. A. V. Duthie.

Tulostoma sp. (a small plant). On ground. A. V. Duthie.

\section{Sphaerioidaceae.}

\section{Deuteromycetes.}

Actinonema rosae (Lib.), Fr., Rosa sp. Nov. 1921.

Ascochyta pisi, Lib., Pisum sativum. Nov. 1921.

Phyllosticta richardiae, Hals., Zantedaschia aethiopica. Oct. 1921

Phyllosticta violae, Desm., Viola odorata. Nov. 1921.

Septoria graminum, Desm., Triticum vulgare. Sept.1921.

Septoria lycopersica, Speg., Solanum lycopersicum. Feb. 1922.

Septoria pelargonii, Syd., Pelargonium sp. C. K. Brain.

Septoria pisi, Went., Pisum sativum.

Septoria rubi, West., Rubus vitifolius. M. M. Radloff. Oct. 1921.

Septoria sp., Silene gallica.

Sphaeropsis malorum, Peck, Cydonia vulgaris ; Pirus malus.

\section{Melanconiaceae.}

Gloeosporium ampelophagum, Sacc., Vitis vinifera. Dec. 1921.

Pestalozia sp., Pirus malus. Oct. 1921.

Septogloeum arachidis, Rac., Arachis hypogea. Feb. 1922.

Septogloeum mori, Lév., Morus sp. March 1922.

\section{Monitiaceae.}

Botrytis cinerea, Pers., Vitis vinifera. March 1921.

Botrytis sp., Lathŷrus odorata.

Oidiopsis taurica, Lév., Tropaeolum major. Feb. 1922.

Oidium sp., Euphorbia sp. Nov. 1921.

Oidium sp., Quercus sp. Nov. 1921. 


\section{Dematiaceae.}

Alternaria solani (E. \& M.), Jones \& Grout, Solanum tuberosum, Nov. 1921. Solanum lycopersici, Feb. 1922.

Cercospora protearum, Cke., Leucospermum conocarpum.

Cladosporium zeae, Peck., Zea Mays. Feb. 1922.

Coniothecium macowanii, Sacc., Protea grandiflora. June 1913.

Macrosporium cladosporioides, Desm., Beta vulgaris. Feb. 1922.

Macrosporium iridis, C. \& E., Iris sp. Feb. 1922.

Macrosporium maydis, C. \& E., Zea Mays. Feb. 1922

Macrosporium sp. Tree tomato. Dec. 1921.

Macrosporium sp., Eriobotryon japonica. Feb. 1922.

Macrosporium sp., Populus sp. March 1922.

\section{Mucedinaceae.}

Rhinotrichum rubiginosum, Fr. A. V. Duthie.

\section{Stilbaceae.}

Isaria sp., Gonipterus scutellatus. C. K. Brain.

\section{Mycelium Sterile.}

Rhizoctonia, Solanum tuberosum. 


\section{$2 \mathrm{BHL}$ Biodiversity Heritage Library}

Van der Byl, P.

A.

$\uparrow$

. 1922. "FUNGI OF THE STELLENBOSCH DISTRICT AND IMMEDIATE VICINITY."

Transactions of the Royal Society of South Africa 10, 281-288.

https://doi.org/10.1080/00359192209519288.

View This Item Online: https://www.biodiversitylibrary.org/item/182861

DOI: https://doi.org/10.1080/00359192209519288

Permalink: https://www.biodiversitylibrary.org/partpdf/175646

\section{Holding Institution}

Smithsonian Libraries

\section{Sponsored by}

Biodiversity Heritage Library

\section{Copyright \& Reuse}

Copyright Status: Not in copyright. The BHL knows of no copyright restrictions on this item.

This document was created from content at the Biodiversity Heritage Library, the world's largest open access digital library for biodiversity literature and archives. Visit BHL at https://www.biodiversitylibrary.org. 